
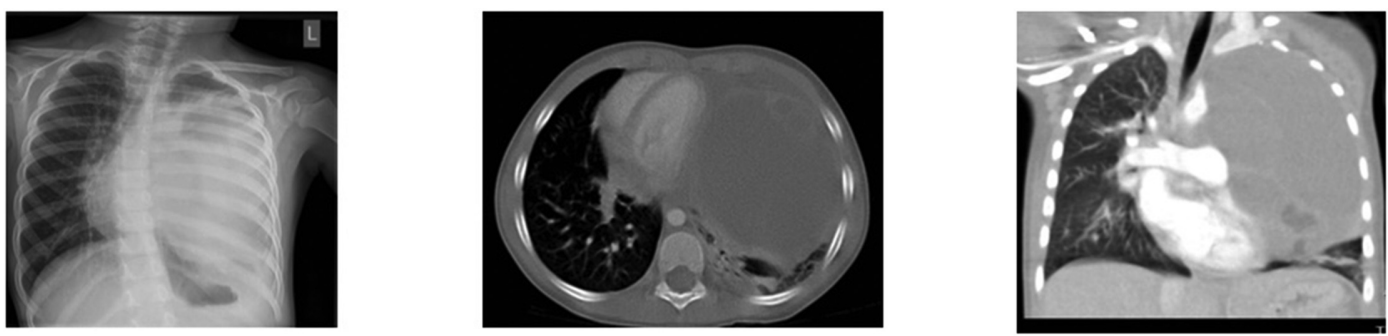

Abstract G326(P) Figure 3a CXR shwowing extensive opacity in the left hemithorax causing mediastinal shift Abstract G326(P) Figure 3b A CT scan showing a large cystic mass Abstract G326(P) Figure 4a CT scan showing large cystic mass

\section{G327(P) USING REGULAR AUDIT TO DEMONSTRATE IMPROVEMENTS IN PAEDIATRIC AND NEONATAL PRESCRIBING}

${ }^{1}$ AT Tsyben, ${ }^{2}$ WK Kelsall, ${ }^{3} \mathrm{NG}$ Gooding. 'School of Clinical Medicine, University of Cambridge, Cambridge, UK; ${ }^{2}$ Department of Paediatrics, Addenbrooke's Hospital, Cambridge, UK; ${ }^{3}$ Central Pharmacy Department, Addenbrooke's Hospital, Cambridge, UK

\subsection{6/archdischild-2015-308599.303}

Aims Prescribing audits have previously shown that the Women's and Children's Directorate reported higher numbers of prescription errors than other areas in the Trust. The directorate introduced a number of initiatives to improve the quality of prescribing and patient safety. A prescribing team (PT), including senior clinicians, pharmacists and trainees, was formed to monitor progress. Strategies included improving departmental induction, establishing designated prescribing areas and reviewing all errors with the prescriber. Six monthly audits have been conducted to review the quality of prescribing. The impact of these initiatives on paediatric prescribing was assessed.

Methods All inpatient drug charts across the paediatric and neonatal wards were reviewed on three non-consecutive days over a period of three weeks. Prescribing errors were identified by the ward pharmacist. Errors were grouped according to type and further analysed by the PT. Errors deemed to have no clinical significance were excluded. Error rates were compared to the previous audits performed with identical methodology.

Results There were 174 (14\%) errors out of 1225 prescriptions on 181 drug charts, an overall reduction of $2 \%$ from the last audit (autumn 2013). Improvements achieved were: 24\% reduction in drug name errors (21); 6\% decrease in dosing errors (23); $11 \%$ less errors in strength of preparation (6); $17 \%$ improvement in charting allergies (10 omissions). All charts included patient weight. Prescriber's signature omission occurred in $11(5 \%)$ prescriptions with no improvement from the previous audit. The number of drug charts that contained five or more errors was 6 out of 181 charts representing a decrease of $2 \%$ since last audit. Disappointingly, there was no improvement in the number of charts containing no errors (84 (47\%)).

Conclusion Decreases in the number of prescription errors suggests that the initiatives introduced by the department continue to impact the prescription standards. The introduction of an electronic prescribing system should potentially reduce errors further, standardising drug names and eliminating signature and allergy omissions. Future work will be required to assess the impact of electronic systems on prescribing.

\section{G328(P) EVALUATION OF MEDICAL STUDENT PERCEPTION OF PERFORMANCE OF A TASK VERSUS THE ACTUAL PERFORMANCE}

${ }^{1} G$ Davison, ${ }^{2} \mathrm{D}$ O'Donoghue, ' $\mathrm{L}$ Hanna, ${ }^{2} \mathrm{~T}$ Dornan, ${ }^{2} \mathrm{M}$ Stevenson, ${ }^{1} \mathrm{~A}$ Thompson. ${ }^{1}$ Paediatrics, Royal Belfast Hospital for Sick Children, Belfast, UK; ${ }^{2}$ Queens University Belfast, Belfast, UK

\subsection{6/archdischild-2015-308599.304}

Aims Approximately $2404^{\text {th }}$ year medical students from Queen's University Belfast rotate through paediatric units in N. Ireland each year. Paediatric Objective Structured Clinical Examinations revealed poor performance at prescribing paediatric medication despite attendance at an Interprofessional Pharmacy Workshop. The aim of this study is to assess perception of task performance, assess actual task performance and compare. Methods An end of attachment assessment was carried out on 85 students. Students completed a 'Paediatric Skills Survey' form, indicating on a 5 point likert scale their perceived competency at 16 various tasks. Students were subsequently assessed on three reciprocal tasks, which included prescribing common paediatric medication, prescribing paediatric intravenous maintenance fluids and plotting growth parameters on an appropriate centile chart. Tasks were marked based on pre-defined criteria and results were subsequently analysed in comparison with perception.

Results Results for perception versus performance of skill performance is shown in Figures 1 and 2 respectively. Statistical analysis of perception in respect to actuality gave kappa values $-0.010,-0.024$ and 0.021 for medication prescription, growth

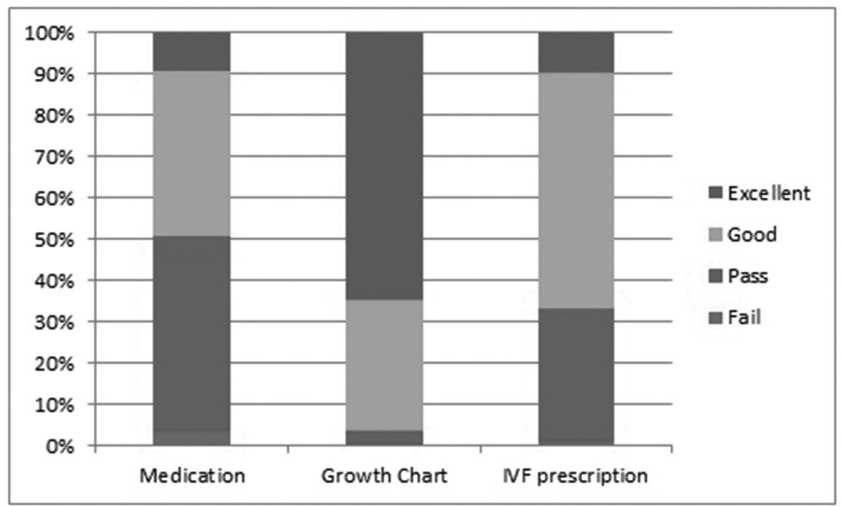

Abstract G328(P) Figure 1 Perception of performance 


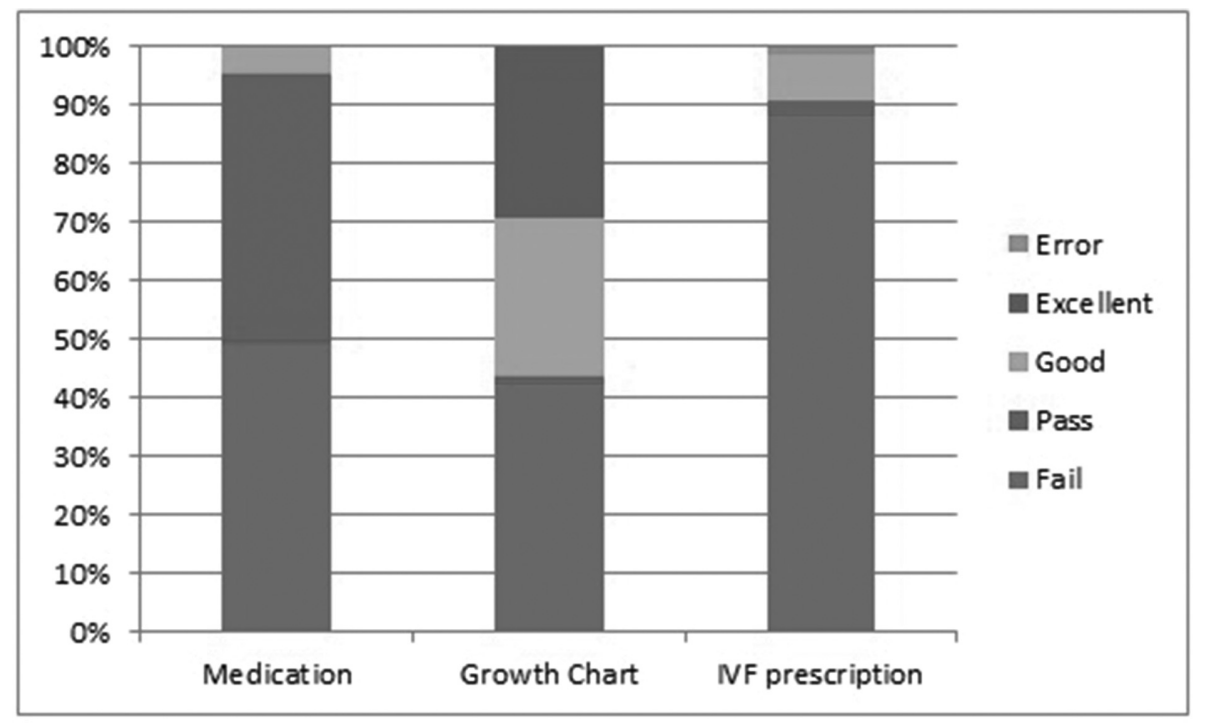

Abstract G328(P) Figure 2 Actual performance

chart plotting and intravenous fluid prescription respectively. All confidence intervals contained zero. Therefore, we are unable to regret the null hypothesis that there is no correlation between perception and performance. The Sign Test is highly significant in all 3 areas, with $\mathrm{P}<0.001$, which indicates clear bias in the direction of more optimistic expectations than reality.

Conclusion The assumption that students are able to indicate assurances in skill performance is disproven. Lack of correlation between perception and performance would put a greater emphasis on continued assessment of medical students and doctors in training.

\section{G329(P) TRIAL OF A HANDHELD VEIN ILLUMINATOR IN AN ACUTE PAEDIATRIC DEPARTMENT}

EM Strehle, S Kaura. Child Health, Northumbria Healthcare Trust, North Shields, UK

\subsection{6/archdischild-2015-308599.305}

Aim Invasive procedures including venepuncture and venous cannulation are distressing experiences for ill children. Painful past experiences and needle phobia can cause the procedure to be more traumatic for the child and more difficult for healthcare professionals. Devices using near infra-red spectroscopy can aid the accurate location of superficial veins thus reducing the number of attempts and time taken to undertake these procedures, and aim to reduce the stress for all involved. The purpose of this study was to evaluate the usefulness and acceptability of a commercially available portable vein illuminator in two acute paediatric departments.

Methods A range of health professionals used the device for venepuncture and venous cannulation in children presenting with acute or chronic illness to the departments. A structured questionnaire was completed by the user once the procedure was completed.

Results 40 patients were enrolled in this study (males $=23$, females $=17$, mean age 4.8 years). Thirty six children underwent venepuncture and 4 underwent venous cannulation. The procedure was completed by registrar in 4 children, by a senior house officer in 24 children and by a nurse in 12 children. Sixty five per cent found the visibility of the veins improved, 5\% found the visibility worse, and 30\% were neutral. The device was found to be very acceptable or acceptable to parents and children in $92.5 \%$ and was graded very useful or useful in $65 \%$ and not useful in $35 \%$.

Conclusion The device was found to be useful and acceptable and did aid with phlebotomy within the departments. The best results were seen in younger children whose superficial veins were not visible to the naked eye. Further studies are required with a larger sample size including a wide range of ethnic groups, and ideally with a comparison group.

\section{G330(P) THE INTRODUCTION OF A REGULAR PRESCRIBING AUDIT AND TRAINING SESSION FOR PAEDIATRIC DOCTORS}

S Ghazal, E Bayles, B Shillitoe, G Nyamgunduru. Paediatric Department, University Hospital of North Durham, Durham, UK

\subsection{6/archdischild-2015-308599.306}

Background A recent UK study showed that the prevalence of medication errors in children could be as high as $13.2 \%$ compared to a previously documented range of $2-14 \%$. To tackle this, a pharmacist led session for all new junior doctors rotating into paediatrics was instigated in a DGH to raise awareness of the issues associated with prescribing in children.

Aim To implement prescribing audit and training sessions to all grade of paediatric medical staff.

Method To further evaluate and enhance the impact of the induction training, an audit was developed based upon the Trust Medicines Management ten prescribing standards. All doctors in attendance at a regular training meeting were split into two groups, each with a mix of grade of staff. Each group evaluated five drug charts taken from the inpatient ward, against the Trust standards. Results were fed back immediately using computer software with time for reflection and discussion followed by a presentation regarding the implications of poor prescribing. Feedback was sought from participants following the session. The audit was carried out twice during the junior rotation, to identify improvements and concerns. 